\title{
Neoantigen: A New Breakthrough in Tumor Immunotherapy
}

\author{
Zheying Zhang ${ }^{1}$, Manman $\mathrm{Lu}^{1}$, Yu Qin ${ }^{1}$, Wuji Gao ${ }^{1}, \mathrm{Li} \mathrm{TaO}^{2}$, Wei Su${ }^{3}$ and Jiateng Zhong ${ }^{1 \star}$ \\ 1 Department of Pathology, Xinxiang Medical University, Xinxiang, China, ${ }^{2}$ Department of Gastroenterology, Cancer Hospital \\ Affiliated to Zhengzhou University, Zhengzhou, China, ${ }^{3}$ Department of Pathology, The First Affiliated Hospital of Xinxiang \\ Medical University, Xinxiang, China
}

OPEN ACCESS

Edited by:

Xiangqian Guo,

Henan University, China

Reviewed by:

Xiangpeng Dai,

Jilin University, China

Jia Liu,

Qingdao University, China

${ }^{*}$ Correspondence: Jiateng Zhong

jtzhong@xxmu.edu.cn

Specialty section: This article was submitted to

Cancer Immunity and Immunotherapy,

a section of the journal

Frontiers in Immunology

Received: 25 February 2021

Accepted: 30 March 2021

Published: 16 April 2021

Citation:

Zhang Z, Lu M, Qin Y, Gao W, Tao L, Su W and Zhong J (2021) Neoantigen: A New Breakthrough in Tumor Immunotherapy.

Front. Immunol. 12:672356. doi: 10.3389/fimmu.2021.672356
Cancer immunotherapy works by stimulating and strengthening the body's anti-tumor immune response to eliminate cancer cells. Over the past few decades, immunotherapy has shown remarkable efficacy in the treatment of cancer, particularly the success of immune checkpoint blockade targeting CTLA-4, PD-1 and PDL1, which has led to a breakthrough in tumor immunotherapy. Tumor neoantigens, a new approach to tumor immunotherapy, include antigens produced by tumor viruses integrated into the genome and antigens produced by mutant proteins, which are abundantly expressed only in tumor cells and have strong immunogenicity and tumor heterogeneity. A growing number of studies have highlighted the relationship between neoantigens and T cells' recognition of cancer cells. Vaccines developed against neoantigens are now being used in clinical trials in various solid tumors. In this review, we summarized the latest advances in the classification of immunotherapy and the process of classification, identification and synthesis of tumor-specific neoantigens, as well as their role in current cancer immunotherapy. Finally, the application prospects and existing problems of neoantigens were discussed.

Keywords: immunotherapy, neoantigen, tumor-specific antigens, vaccine, personalized cancer immunotherapy

\section{INTRODUCTION}

The human immune system helps us avoid infections and many diseases and protects us from cancer $(1,2)$. With the ability to recognize its own and non-self substances, the body's immune system can produce natural immune tolerance to its own components and eliminate non-self foreign bodies to maintain the internal environment's stability (3). Cancer occurs when normal cells change and begin to lose control. Since cancer cells are derived from normal cells and are indistinguishable from normal cells, the immune system's ability to recognize cancer cells is minimal $(4,5)$. Cancer cells can avoid being attacked by the immune system when the immune system mistakenly thinks tumor cells are self-components. The surveillance of the immune system is also progressively weakened by mutations in the tumor. Tumor cells that activate the immune system are gradually screened out until they produce tumor molecules that are not recognized by the immune system. This process is also known as immunoediting of tumor. In this way, tumor cells successfully escape the damage of the immune system and have a chance to develop. What's more, because cancer cells themselves can also release many substances that block the immune system, tumor immune response is often selectively suppressed around the tumor tissue $(6,7)$, which 
explains the ineffectiveness of immunotherapy in many patients: it is the failure to activate the immune response around the tumor tissue rather than the inability to activate the immune response systematically (6-9). In addition, inflammation can promote the development of tumors. Inflammation can release a large number of immunosuppressive cytokines locally in tumor tissue and suppress the immune system through a variety of ways. So cancer still could be caused even with a normal immune system. To overcome this problem, researchers have been looking for ways to help the immune system enhance its antitumor immune responses and improve its capacity to suppress tumor. In recent years, immunotherapy has developed rapidly and become a mature cancer treatment strategy in addition to surgery, chemotherapy and radiotherapy. Immunotherapy has shown a significant therapeutic effect in many human malignant tumors by using the immune system to eliminate cancer cells (10).

With the wide application of high-throughput omics and the development of neoantigen prediction technology, immunotherapy based on neoantigen has become a new research hotspot. Neoantigens are mainly tumor-specific antigens generated by mutations in tumor cells, which are only expressed in tumor cells (11). Neoantigens can also be produced by viral infection, alternative splicing and gene rearrangement (12-14). They are ideal targets for T cells to recognize cancer cells and can stimulate strong anti-tumor immune response. Studies in the past five years have shown that neoantigens play a key role in tumor immunotherapy. The identification, screening and identification of neoantigens accelerate the development of personalized immunotherapy for tumor patients, which will benefit more patients (15). As more scientific and clinical data reveal the remarkable effects of neoantigen-based vaccine therapies in a variety of cancer types, there is ample reason to believe that neoantigen-based therapies will be a promising area of cancer immunotherapy.

\section{INTRODUCTION OF CANCER IMMUNOTHERAPY}

Immunotherapy refers to the measures taken to use immunological methods and principles to target the hyper or hypo-immune state of the organism, intervene or adjust the organism's immune function artificially, and strengthen or attenuate the immune response so as to achieve the purpose of treating diseases (16). Immunotherapy enhances the immune system's ability to recognize, target, and eliminate cancer cells in the body, making it a potentially universal cancer solution (17). Immunotherapy, approved as a first-line treatment strategy for multiple cancers in the United States and elsewhere (18), can be used alone or in combination with other cancer treatments (19). Compared with other cancer treatments, immunotherapy has become more precise, personalized, and has fewer side effects (20-22). In recent years, it has gradually become an important development direction of cancer treatment, which is known as the fourth leading cancer treatment technology after surgery, radiotherapy, and chemotherapy.

\section{CLASSIFICATION OF IMMUNOTHERAPIES}

Immunotherapy is generally divided into two categories: active immunotherapy and passive immunotherapy (23) (Figure 1). Active immunotherapy refers to eliminating cancer cells by stimulating the body's immune system (24). Passive immunotherapy refers to the passive acceptance by an organism of antibodies, cytokines, or transformed immune cells that can directly act on the tumor (25).

Tumor vaccines, one type of active immunotherapy, is an important component in the field of tumor immunotherapy (26). Tumor vaccines can recognize proteins present on specific cancer cells, arrest cancer cell growth, prevent cancer

\section{Classification of Immunotherapies}

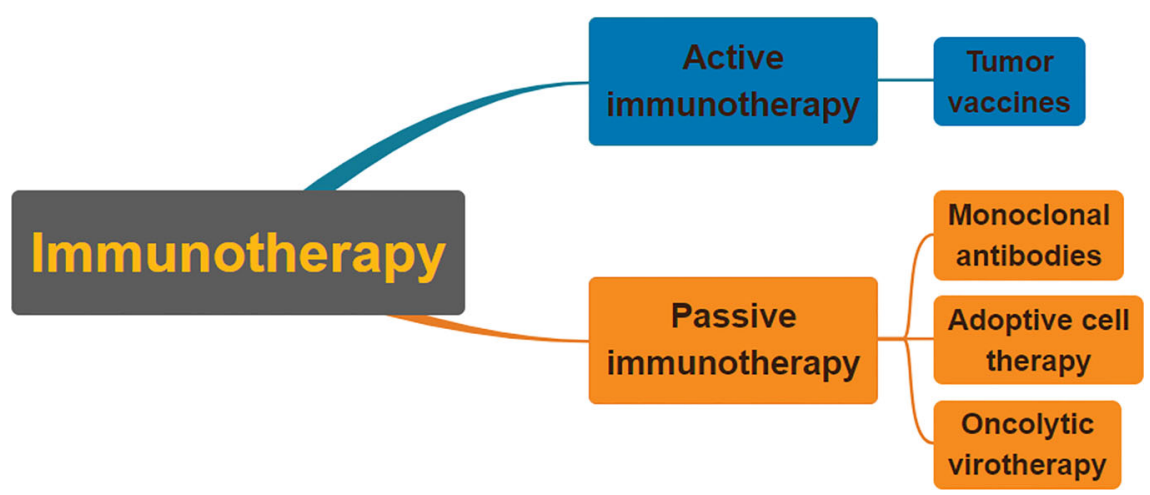

FIGURE 1 | Classification of immunotherapies. Immunotherapy is usually divided into passive immunotherapy and active immunotherapy. Active immunotherapy is mainly cancer vaccines. Passive immunotherapy mainly includes adoptive cell therapy, oncolytic viruses, and monoclonal antibodies. 
recurrence, and clear cancer cells that remain after treatment (27-30). Cancer vaccines aim to elicit immune responses against tumor-specific or tumor-associated antigens, thereby facilitating the immune system to attack cancer cells carrying these antigens $(30,31)$. Tumor vaccines include cell vaccines, DNA vaccines, mRNA vaccines, polypeptide vaccines, dendritic cell vaccines, and others (32, 33). In April 2010, Provenge (sipuleucel-T), the first tumor therapeutic vaccine, was approved by the FDA for the treatment of prostate cancer (34).

Monoclonal antibodies have promising therapeutic effects in the clinic and belong to passive immunotherapy (35). Monoclonal antibody drugs can specifically bind to specific receptors or ligands on the surface of tumor cells or immune cells and block the corresponding signaling pathways, thereby exerting antitumor effects (36). The hottest monoclonal antibodies in the field of oncology at present are immune checkpoint inhibitors $(37,38)$. Immune checkpoints are immunosuppressive pathways that they can suppress T-cell activity $(39,40)$, and cancer cells evade the immune response by hijacking this pathway (41-44). The most thoroughly studied immune checkpoints are CTLA-4, PD-1, and PD-L1, which have been approved by FDA for the treatment of a variety of tumors $(45,46)$. Adoptive cell therapy exerts its tumorsuppressive and killing effects by isolating immunocompetent cells from patients, inducing their differentiation in vitro, reconstituting, expanding, and re-infusing them into patients to target antigen-specific tumor cells and exert their tumorsuppressive and tumor-killing effects (47). It belongs to passive immunotherapy (48). The most promising adoptive cell therapies at present are TCR-T(TCR-modified T cell) and CAR-T(Chimeric antigen receptor T cell) (49-51). Oncolytic virotherapy is a form of passive immunotherapy. Oncolytic viruses are a class of tumorkilling type viruses that render them unable to replicate in normal tissues by attenuating or deleting viral pathogenic factors while maintaining replication and killing viability in tumor cells $(52,53)$. IMLYGIC, the first FDA-approved oncolytic viral drug, was genetically modified from herpes virus type 1 (HSV-1) for the treatment of metastatic melanoma (54).

There are two current successes of immunotherapy, one with PD-1 and PD-L1 mAbs derived through immune checkpoints and the other with adoptive $\mathrm{T}$ cell therapy $(29,55)$. But clinical trials have shown that although having promising potential, immune checkpoint therapies have limited efficacy in many cases, especially in solid tumors with low response rates (56). What's more, adoptive $\mathrm{T}$ cells present problems such as poor persistence as well as cytotoxicity in vivo and can trigger an inflammatory factor storm (57-59). With the wide application of multi-omics high-throughput technologies and the development of neoantigen prediction technology, neoantigen-based immunotherapy becomes a new research hotspot. In the past five years, studies have shown that neoantigens have a promising outcome in clinical therapy. Technological advances in the identification, screening, and characterization of neoantigens will accelerate the development of individual immunotherapy for cancer patients, thereby benefiting more patients. In the future, neoantigen therapy will become an important therapeutic modality in the field of precision oncology (60).

\section{WHAT IS THE NEOANTIGEN?}

Gene mutations caused by genetic instability during carcinogenesis always occur in the non-coding and coding region, and the amino acid sequence changes caused by mutations in the coding region can produce proteins that are not found in normal cells. These proteins can activate the immune system and lead to the immune system's attack on cancer cells (61). Neoantigens can also be produced by viral infection, alternative splicing and gene rearrangement. These aberrant antigens, which can be recognized by immune cells and result from mutations in cancer cell genes, are neoantigen (62). Neoantigens can be presented on the cell surface and subsequently recognized by $\mathrm{T}$ cells under the action of major histocompatibility complex (MHC) molecules $(63,64)$. Tumor antigens are divided into tumor associated antigens (TAA) and tumor specific antigen (TSA) (65). TAA is a protein expressed by unmutated genes and appears to be significantly over-expressed in tumor cells but rarely expressed in normal cells (11). Because TAAs are normal host proteins, they are subject to both central and peripheral tolerance mechanisms $(35,64)$. Targeting TAAs may also lead to autoimmune toxicity (39); tumor specific antigen (TSA) is a neoantigen resulting from somatic mutations and is expressed only in tumor cells but not in normal cells (66). Because normal cells do not express TSA, they are considered non-self by the immune system, neoantigen specific immune responses are not affected by tolerance. Furthermore, targeting TSAs does not easily induce autoimmunity (39). Thus, neoantigens are ideal targets for therapeutic cancer vaccines and $\mathrm{T}$ cell-based cancer immunotherapy. By taking advantage of the immune activity of neoantigens, synthetic neoantigen drugs can be designed according to the situation of tumor cell mutation to achieve the effect of treatment.

\section{CLASSIFICATION OF NEOANTIGENS}

Neoantigens can be classified into two categories: shared neoantigens and personalized neoantigens $(66,67)$ (Figure 2). Shared neoantigens refer to mutated antigens that are common across different cancer patients and not present in the normal genome. Shared neoantigens that are highly immunogenic have the potential to be screened for use as broad-spectrum therapeutic cancer vaccines for patients with the same mutated gene $(68,69)$. Personalized neoantigens refer to mutated antigens that are unique to most neoantigens and completely different from patient to patient. Thus, the personalized neoantigen preparation drug can only be specifically targeted to each patient, that is, personalized therapy (70). Neoantigens, with strong immunogenicity, can reduces the probability of immune escape of tumor cells. However, the different types and quantities of neoantigens in different individuals of the same tumor caused by specificity of mutations showing obvious individual heterogeneity. Therefore, the application of neoantigens in tumor immunotherapy will tend to be personalized (71). Individualized cancer vaccines can work alone or in combination with other therapies to increase the strength and 


\section{Classification of neoantigens}
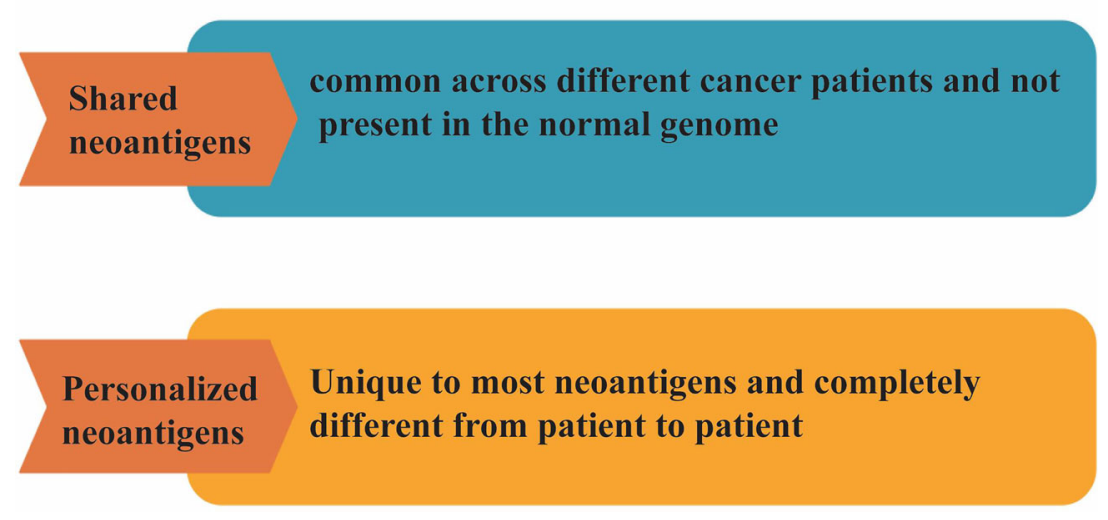

FIGURE 2 | Classification of neoantigens. Neoantigens can be classified into two categories: shared neoantigens and personalized neoantigens. Shared neoantigens refer to mutated antigens that are common across different cancer patients and not present in the normal genome. Personalized neoantigens refer to mutated antigens that are unique to most neoantigens and completely different from patient to patient.

durability of the anti-tumor effect, improve survival and quality of life, and ultimately improve the outcome of cancer treatment for patients (72). The feasibility, safety and immunogenicity of individualized cancer vaccine in the treatment of cancer patients determine that it will be an important development trend in the future (15). It is expected that individualized cancer vaccines will enable most patients to obtain precise treatment in the foreseeable future.

\section{IDENTIFICATION OF NEOANTIGENS}

Although neoantigens have made good clinical progress in tumor therapy, the number of neoantigens with immunogenicity is small, and the prediction comparison is difficult (73). Therefore, the neoantigen field needs more optimized algorithms and validated methods for accurate prediction in order to select more reliable neoepitopes of high immunogenicity. At present, the prediction accuracy of tumor neoantigen remains an urgent problem. For tumor neoantigen prediction algorithm, there are many factors to be considered, including HLA typing, expression, mutation analysis, prediction peptide processing, TCR binding force, MHC affinity, PMHC stability, tumor neoantigen source,and so on $(74,75)$. It also includes $\mathrm{T}$ cell recognition, TCR analysis, and immune cell analysis to assess $\mathrm{T}$ cell response $(74,76)$. For neoantigen screening and assessment of $\mathrm{T}$ cell responses, in addition to next-generation sequencing, there are high-resolution and tandem mass spectrometry techniques as well as in silico techniques for peptide prediction, but prediction algorithms based on machine learning and AI techniques need to be continuously trained with confirmatory datasets where data type, quality, and quantity can greatly affect algorithm precision $(77,78)$ (Figure 3 ). Therefore, the continuous accumulation of databases, especially the validated tumor neoantigen data, is extremely critical to improving algorithm accuracy $(79,80)$. The tumor neoantigen selection Alliance (TESLA) was initiated and formed by the Park Institute for cancer immunotherapy (PICI) and the Cancer Research Institute (CRI) (81). TESLA brings together 36 top biotechnology, pharmaceutical, university, and non-profit research teams, consisting of the National Cancer Center (NCC), PICI, Memorial Sloan-Kettering Cancer Center (MSKCC), MD Anderson Cancer Center, and more than 30 other top neoantigen research institutions. The consortium aims to establish algorithms and standards for global neoantigen testing, make concerted efforts to predict more precise anticancer targets, and advance research and application of personalized tumor vaccines. TESLA scientists discovered algorithmic models and core parameters that can better predict neoantigens and accurately predicted $75 \%$ of validated neoantigen targets and filtered to exclude $98 \%$ of invalid neoantigen targets, whose findings were published in cell journals (81).

\section{NEOANTIGEN SYNTHESIS PROCESS}

The development strategy for tumor neoantigen vaccines is clear. First, obtaining tumor tissue and normal tissue samples from the patient and then identifying the mutant genes of the tumor by comparing the exome sequencing results of the two groups of samples. cDNA microarray or RNA sequence test was performed to select appropriate mutated neoantigens according to gene expression level. Computer analysis is used to predict the adhesion affinity of candidate antigens to HLA, and the gene sequences that are most likely to become neoantigens of tumors are screened. Finally, these mutated genes are designed into vaccines $(82,83)$. Cancer vaccines can come in many forms, such as peptide vaccines, dendritic cell vaccines, mRNA vaccines, DNA vaccines, and viral vaccines (84) (Figure 4). Different forms of vaccines have different advantages and disadvantages (Table $\mathbf{1}$ ). 


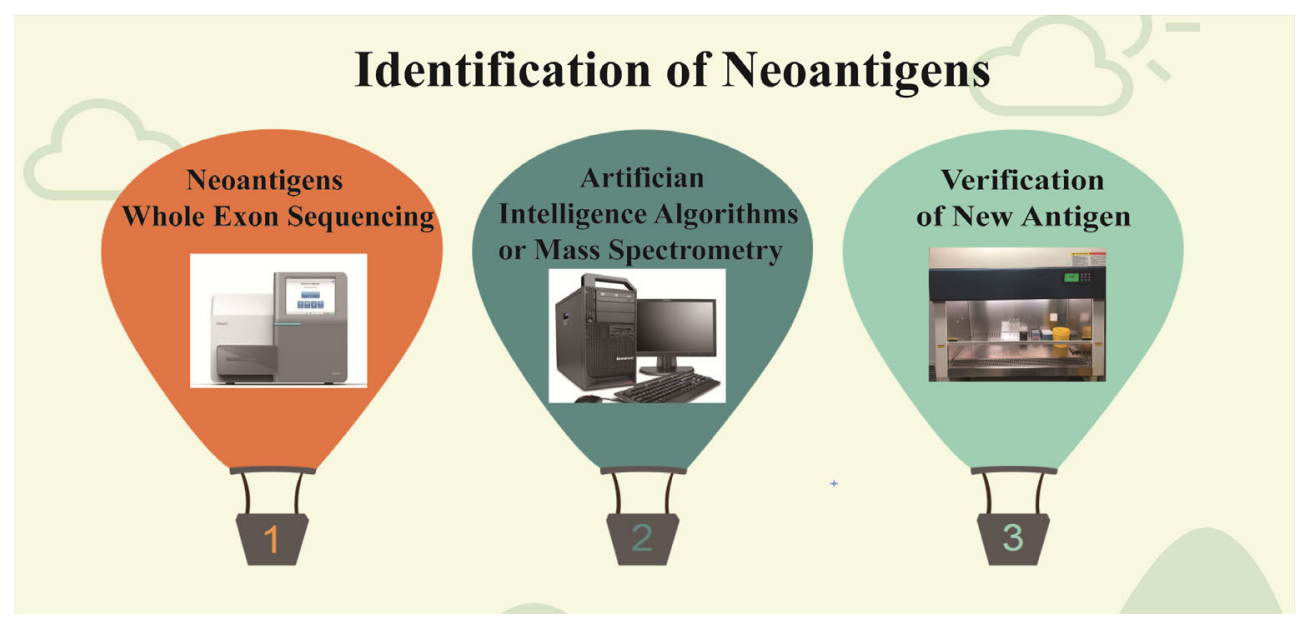

FIGURE 3 | Identification of neoantigens. First, the new antigens were found by high-throughput sequencing, then screened by algorithm and mass spectrometry, and finally verified by experiment.

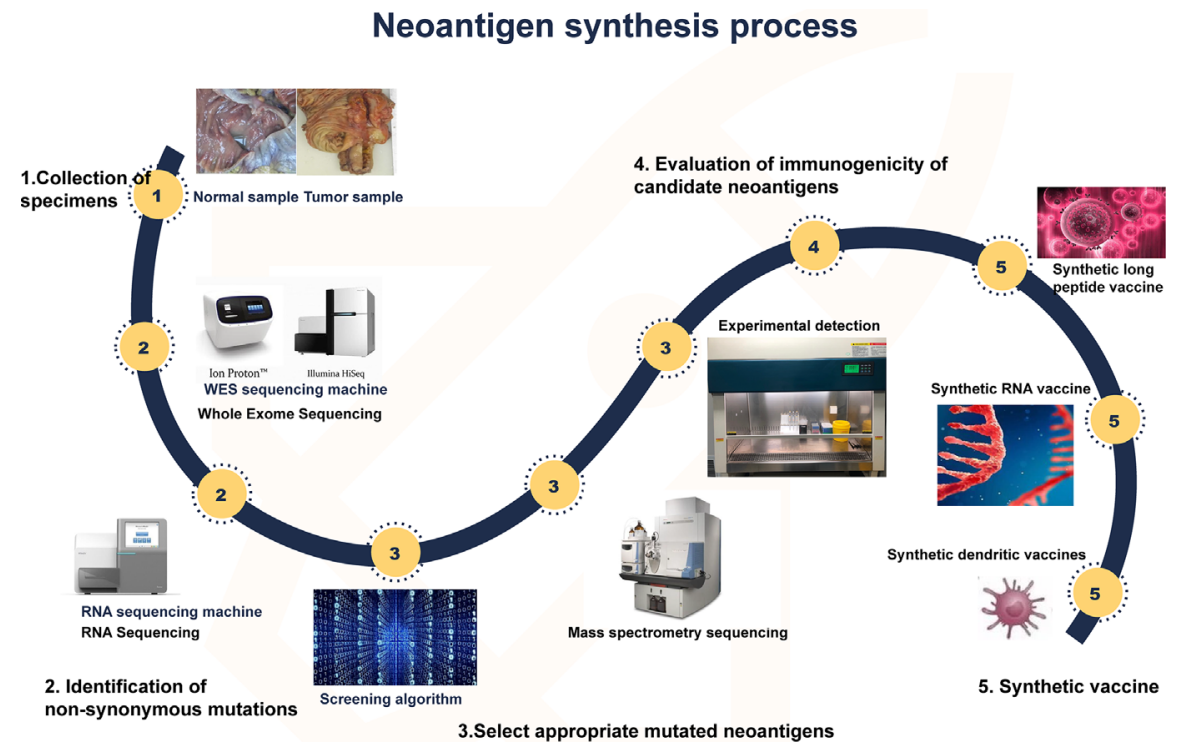

FIGURE 4 | Synthesis of neoantigen. First, tumor and normal tissue samples were obtained. Then, by comparing the sequencing results of the two groups of samples, the mutated gene of the tumor was identified. Using computer, mass spectrometry or experimental methods to screen the gene sequences that are most likely to become tumor neoantigens, and finally these mutated genes can be designed into vaccines, which can take many forms, such as peptide vaccines, dendritic cell vaccines, RNA vaccines, etc.

\section{ADVANCES IN THE STUDY OF NEOANTIGENS}

In 2014, Rosenberg reported in Science that he had successfully treated a patient with advanced cholangiocarcinoma, a highly aggressive form of the disease, using lymphocytes that were amplified in vitro and specifically recognized cancer cells (85). Whole exome sequencing revealed a total of 26 significant gene mutations in the patient, many of which produced abnormal proteins. By co-culturing these abnormal protein fragments with lymphocytes isolated from the patient's tumor tissue, CD4 positive $\mathrm{T}$ cells were found to recognize one abnormal protein, ERBB2IP. Lymphocytes that recognize the aberrant protein produced by the ERBB2IP mutation were expanded, activated, and returned to the patient. After two regurgitation, the lesion shrank significantly throughout the patient's body, leading to complete remission and complete disappearance of the tumor. Cholangiocarcinoma is an extremely diffificult disease, and this 
TABLE 1 | Advantages and disadvantages of different forms of vaccines.

\begin{tabular}{|c|c|c|}
\hline $\begin{array}{l}\text { Vaccines } \\
\text { type }\end{array}$ & Advantages & Disadvantages \\
\hline $\begin{array}{l}\text { mRNA } \\
\text { vaccines }\end{array}$ & $\begin{array}{l}\text { 1. Cellless production; } \\
\text { 2. Delivered to DC efficiently }\end{array}$ & Easy degradation \\
\hline DNA vaccines & $\begin{array}{l}\text { 1. Celless production; } \\
\text { 2. The cost is low; } \\
\text { 3. Encode any epitope }\end{array}$ & There is a risk of mutation \\
\hline $\begin{array}{l}\text { Peptide } \\
\text { vaccines }\end{array}$ & $\begin{array}{l}\text { 1. Cellless production; } \\
\text { 2. Easy to transport; } \\
\text { 3. Fully degradable }\end{array}$ & $\begin{array}{l}\text { Degradation produces an } \\
\text { unrelated immune response }\end{array}$ \\
\hline $\begin{array}{l}\text { Dendritic cell } \\
\text { vaccines }\end{array}$ & $\begin{array}{l}\text { 1. The immune stimulation } \\
\text { activity is strong; } \\
\text { 2. The clinical effect has } \\
\text { been proved }\end{array}$ & Cost is high \\
\hline
\end{tabular}

patient achieved complete remission with lymphocyte infusion for several years after the failure of conventional treatments, such as chemotherapy. This report foreshadows the future role of neoantigens in immunotherapy. In 2015, Rosenberg team also reported the discovery of multiple neoantigens in the tissues of patients with digestive tract tumors, including the famous neoantigen derived from KRAS (G12D) mutation (86).

At present, a large number of neoantigens have been discovered, which are unique to cancer cells and recognized by $\mathrm{T}$ cells as heterogeneous, and are not affected by immune tolerance mechanism. Therefore, these antigens can be used as effective targets for immune-mediated tumor therapy. In 2017, Ugur Sahin's team reported in Nature that a personalized RNAbased vaccine treatment regimen was used in a trial involving 13 melanoma patients. The therapy predicted the antigen of the mutations detected on each patient's genome and then synthesized a personalized vaccine. Eight of these patients have had no further tumor recurrence over 23 months, five of them with advanced malignant melanoma, two of whom experienced significant tumor shrinkage, and one additional patient treated with the vaccine combined with a PD1 antibody had a complete tumor response. This study demonstrated the clinical feasibility, safety, and antitumor activity of personalized RNA vaccine based on multi-omics (87). In the same year, the vaccine produced by Catherine J. Wu's team using Neoantigen also successfully treated malignant melanoma. Four of the six patients who received the vaccine were free of relapse 25 months after the vaccine, while two patients with recurrent disease were subsequently treated with anti-PD-1 therapy and experienced complete tumor regression (88). Personalized vaccines may be an important way to conquer cancer.

Genocea Biosciences's GEN-009 vaccine trial (NCT03633110) is one of the few Phase 1/2a clinical trials that have shown the best efficacy among the current neoantigen personalization therapies with an estimated completion date of December 2022. The purpose of this study was to evaluate the safety, tolerability, immunogenicity, and antitumor activity of the personalized vaccine GEN-009 for the treatment of patients with solid tumors, which is targeted at a broad range of cancers. The results so far show that 40 doses of the vaccine have been administered, and only a few patients have experienced mild local discomfort caused by the vaccine adjuvant with no dose limited toxicity (DLT) occurred. Ninety-nine percent of the peptides selected for the vaccine produce an immune response, and so far, no patients who have received the vaccine have relapsed $(73,89)$.

In 2019, Patrick Ott reported in the journal Cell the results of a personalized neoantigen vaccine NEO-PV-01 combined with PD-1 blockade in patients with advanced melanoma, non-small cell lung cancer, or bladder cancer. No treatment-related serious adverse events were observed in 82 patients, and $\mathrm{T}$ cell responses were observed in all patients, with no obvious toxic and adverse reactions (90).

A novel bispecific-specific antibody therapy targeting common mutations in TP53 and Ras was reported in Science and Science Immunology in 2021 by Bbert Vogelstein and his team. We all know that TP53 and RAS are important tumorrelated genes in vivo, which are often mutated. However, p53 and Ras are mainly intracellular, so antibody-based therapy cannot be achieved with conventional methods. However, proteins are degraded by the proteasome into peptides, some of which form complexes with human leukocyte antigen (HLA) proteins and are presented on the cell surface. They have developed a specific bispecific single-chain diabody (SCDB) antibody that targets TP53 and RAS mutations. This bispecific antibody can specifically recognize and activate $\mathrm{T}$ cells in vitro and in mice, exerting good anti-tumour effects without cross-reactivity and with a good safety profile $(91,92)$.

We searched ClinicalTrials.gov (https://clinicaltrials.gov/) and found 77 cases of neoantigen studies, many of them have shown the good application value of neoantigen (Search term is "neoantigen"). However, the study of neoantigen therapy starts relatively late and still in the laboratory stage, and there are no related products on the market at both home and abroad.

\section{PROSPECTS AND CHALLENGES}

As the incidence and mortality of cancer continue to increase, people's desire to conquer cancer is more and more urgent. Neoantigen vaccine has shown an obvious effect on tumor treatment in clinical trials and is expected to become an important drug for alleviating the increasing tumor morbidity and mortality in the future. It has attracted the attention of experts in immunotherapy and is a significant development direction in the future. However, there are still some restricting factors in the development of neoantigens, and solving these problems is the key to the widespread popularization of neoantigens.

1. Scarce amount of antigens. Thousands of mutations in nonsynonymous genes are typically found in tumor samples, but only a few ultimately meet the antigen criteria. Finding more effective antigens is a problem that needs to be solved. Studies have shown that most specific antigens tend to be distributed in non-coding regions. The development of non-coding research in recent years will also provide assistance for the discovery of new antigens.

2. Screening methods for predicting neoantigens need to be improved. The lack of effective screening methods for 
neoantigen is another obstacle to the development of neoantigen therapy. At present, the algorithm of predicting neoantigen is in full bloom. With the development of bioinformatics technology, artificial intelligence and machine learning, we believe that this problem will be solved soon.

3. The development cycle of neoantigen vaccine is too long. The long development cycle of neoantigen vaccine is recognized as a primary obstacle to the application of vaccine. The long development cycle leads to the increase of research and development costs, and the great pressure on laboratories and enterprises is not conducive to the clinical application of vaccines. Patients participating in the trial or treatment have a short survival period and a long development cycle, which may lead to some patients being unable to accept the final treatment due to the long drug development cycle.

4. Preparation and delivery of vaccines remains a challenge. Currently, many methods have been developed for the preparation, formulation and delivery of different cancer vaccines. Technically, however, these vaccines need to be manufactured under GMP conditions. The biggest challenge, especially for small nucleic acid therapies such as mRNA/ DNA, is delivery technology.

5. The heterogeneity of the tumor is difficult to resolve. Tumors are heterogeneous in the course of evolution, so each part of the gene that may mutate is different. So it may be a paradox to get local tumor tissue from a patient in the first place to predict the neoantigen of that patient. It is still a difficult

\section{REFERENCES}

1. Janssen LME, Ramsay EE, Logsdon CD, Overwijk WW. The immune system in cancer metastasis: friend or foe? J Immunother Cancer (2017) 5:79. doi: 10.1186/s40425-017-0283-9

2. Lu YC, Wang XJ. Harnessing the power of the immune system in cancer immunotherapy and cancer prevention. Mol Carcinog (2020) 59:675-8. doi: $10.1002 / \mathrm{mc} .23211$

3. Chaplin DD. Overview of the immune response. J Allergy Clin Immunol (2010) 125:S3-23. doi: 10.1016/j.jaci.2009.12.980

4. Sia D, Villanueva A, Friedman SL, Llovet JM. Liver Cancer Cell of Origin, Molecular Class, and Effects on Patient Prognosis. Gastroenterology (2017) 152:745-61. doi: 10.1053/j.gastro.2016.11.048

5. Sanchez-Danes A, Blanpain C. Deciphering the cells of origin of squamous cell carcinomas. Nat Rev Cancer (2018) 18:549-61. doi: 10.1038/s41568-018-0024-5

6. Gonzalez H, Hagerling C, Werb Z. Roles of the immune system in cancer: from tumor initiation to metastatic progression. Genes Dev (2018) 32:126784. doi: $10.1101 / \operatorname{gad} .314617 .118$

7. Baumeister SH, Freeman GJ, Dranoff G, Sharpe AH. Coinhibitory Pathways in Immunotherapy for Cancer. Annu Rev Immunol (2016) 34:539-73. doi: 10.1146/annurev-immunol-032414-112049

8. Pandya PH, Murray ME, Pollok KE, Renbarger JL. The Immune System in Cancer Pathogenesis: Potential Therapeutic Approaches. J Immunol Res (2016) 2016:4273943. doi: 10.1155/2016/4273943

9. Sanmamed MF. Chen L. A Paradigm Shift in Cancer Immunotherapy: From Enhancement to Normalization. Cell (2018) 175:313-26. doi: 10.1016/ j.cell.2018.09.035

10. Waldman AD, Fritz JM. Lenardo MJ. A guide to cancer immunotherapy: from T cell basic science to clinical practice. Nat Rev Immunol (2020) 20:651-68. doi: 10.1038/s41577-020-0306-5

11. Zamora AE, Crawford JC, Thomas PG. Hitting the Target: How T Cells Detect and Eliminate Tumors. J Immunol (2018) 200:392-9. doi: 10.4049/ jimmunol.1701413 problem to use 1-2 specific neoantigens to fully recognize and kill solid tumor tissues.

6. Expensive. Therapies based on neoantigens are mostly personalized, and from initial gene sequencing to validation and production, the cost of treatment can be very high. Cost remains the biggest challenge.

\section{AUTHOR CONTRIBUTIONS}

ZZ has written the review. JZ and WS supervised the program. ML, YQ, WG and TL have discussed and edited the manuscript. All authors contributed to the article and approved the submitted version.

\section{FUNDING}

This work was supported by the National Natural Science Foundation of China (No. U1804173, 81802470 and 81702891), Zhongyuan Qianren Jihua of Henan Province (No. ZYQR201810153), Joint construction project of Henan Medical Science and technology research plan (No. LHGJ20190452), Natural Science Foundation of Henan Province (No. 202300410326), Xinxiang Medical College research funding (No. XYBSKYZZ201632).

12. Zhang Y, Qian J, Gu C, Yang Y. Alternative splicing and cancer: a systematic review. Signal Transduct Target Ther (2021) 6:78. doi: 10.1038/s41392-02100486-7

13. Rathe SK, Popescu FE, Johnson JE, Rathe SK, Popescu FE, Johnson JE, et al. Identification of candidate neoantigens produced by fusion transcripts in human osteosarcomas. Sci Rep (2019) 9:358. doi: 10.1038/s41598-018-36840-z

14. Wang G, Kang X, Chen KS, Jehng T, Jones L, Chen J, et al. An engineered oncolytic virus expressing PD-L1 inhibitors activates tumor neoantigenspecific T cell responses. Nat Commun (2020) 11:1395. doi: 10.1038/s41467020-15229-5

15. Shemesh CS, Hsu JC, Hosseini I, Shen BQ, Rotte A, Twomey P, et al. Personalized Cancer Vaccines: Clinical Landscape, Challenges, and Opportunities. Mol Ther (2021) 29:555-70. doi: 10.1016/j.ymthe.2020.09.038

16. Tan S, Li D, Zhu X. Cancer immunotherapy: Pros, cons and beyond. BioMed Pharmacother (2020) 124:109821. doi: 10.1016/j.biopha.2020.109821

17. Flower KJ, Ghaem-Maghami S, Brown R. Is there a Role for Epigenetic Enhancement of Immunomodulatory Approaches to Cancer Treatment? Curr Cancer Drug Targets (2018) 18:5-15. doi: 10.2174/1568009617666170206105131

18. Barbari C, Fontaine T, Parajuli P, Lamichhane N, Jakubski S, Lamichhane P, et al. Immunotherapies and Combination Strategies for Immuno-Oncology. Int J Mol Sci (2020) 21:5009. doi: 10.3390/ijms21145009

19. Schmidt C. The benefits of immunotherapy combinations. Nature (2017) 552: S67-9. doi: 10.1038/d41586-017-08702-7

20. Wraith DC. The Future of Immunotherapy: A 20-Year Perspective. Front Immunol (2017) 8:1668. doi: 10.3389/fimmu.2017.01668

21. Krzyszczyk P, Acevedo A, Davidoff EJ, Timmins LM, Marrero-Berrios I, Patel $\mathrm{M}$, et al. The growing role of precision and personalized medicine for cancer treatment. Technol (Singap World Sci) (2018) 6:79-100. doi: 10.1142/ S2339547818300020

22. Krzyszczyk P, Acevedo A, Davidoff EJ, Timmins LM, Marrero-Berrios I, Patel $\mathrm{M}$, et al. The growing role of precision and personalized medicine for cancer treatment. Technology (Singap World Sci) (2018) 6:79-100. doi: 10.1142/ S2339547818300020.9 
23. Galluzzi L, Vacchelli E, Bravo-San Pedro JM, Buque A, Senovilla L, Baracco $\mathrm{EE}$, et al. Classification of current anticancer immunotherapies. Oncotarget (2014) 5:12472-508. doi: 10.18632/oncotarget.2998

24. Rodriguez-Cerdeira C, Carnero Gregorio M, Lopez-Barcenas A, SanchezBlanco E, Sanchez-Blanco B, Fabbrocini G, et al. Advances in Immunotherapy for Melanoma: A Comprehensive Review. Mediators Inflammation (2017) 2017:3264217. doi: 10.1155/2017/3264217

25. Baxter D. Active and passive immunization for cancer. Hum Vaccin Immunother (2014) 10:2123-9. doi: 10.4161/hv.29604

26. Chodon T, Koya RC, Odunsi K. Active Immunotherapy of Cancer. Immunol Invest (2015) 44:817-36. doi: 10.3109/08820139.2015.1096684

27. Pan RY, Chung WH, Chu MT, Chen SJ, Chen HC, Zheng L, et al. Recent Development and Clinical Application of Cancer Vaccine: Targeting Neoantigens. J Immunol Res (2018) 2018:4325874. doi: 10.1155/2018/4325874

28. Aurisicchio L, Salvatori E, Lione L, Bandini S, Pallocca M, Maggio R, et al. Poly-specific neoantigen-targeted cancer vaccines delay patient derived tumor growth. J Exp Clin Cancer Res (2019) 38:78. doi: 10.1186/s13046-019-1084-4

29. Le QV, Suh J, Choi JJ, Park GT, Lee JW, Shim G, et al. In Situ NanoadjuvantAssembled Tumor Vaccine for Preventing Long-Term Recurrence. ACS Nano (2019) 13:7442-62. doi: 10.1021/acsnano.9b02071

30. Hollingsworth RE, Jansen K. Turning the corner on therapeutic cancer vaccines. NPJ Vaccines (2019) 4:7. doi: 10.1038/s41541-019-0103-y

31. Tanyi JL, Bobisse S, Ophir E, Tuyaerts S, Roberti A, Genolet R, et al. Personalized cancer vaccine effectively mobilizes antitumor $\mathrm{T}$ cell immunity in ovarian cancer. Sci Transl Med (2018) 10:eaao5931. doi: 10.1126/scitranslmed.aao5931

32. Basu A, Ramamoorthi G, Jia Y, Faughn J, Wiener D, Awshah S, et al. Immunotherapy in breast cancer: Current status and future directions. Adv Cancer Res (2019) 143:295-349. doi: 10.1016/bs.acr.2019.03.006

33. Thomas S, Prendergast GC. Cancer Vaccines: A Brief Overview. Methods Mol Biol (Clifton NJ) (2016) 1403:755-61. doi: 10.1007/978-1-4939-3387-7_43

34. Cheever MA, Higano CS. PROVENGE (Sipuleucel-T) in prostate cancer: the first FDA-approved therapeutic cancer vaccine. Clin Cancer Res (2011) 17:3520-6. doi: 10.1158/1078-0432.CCR-10-3126

35. Pelletier JPR, Mukhtar F. Passive Monoclonal and Polyclonal Antibody Therapies. Immunol Concepts Transfus Med (2020) 16:251-348. doi: 10.1016/B978-0-323-67509-3.00016-0

36. Weiner GJ. Building better monoclonal antibody-based therapeutics. Nat Rev Cancer (2015) 15:361-70. doi: 10.1038/nrc3930

37. Gravbrot N, Gilbert-Gard K, Mehta P, Ghotmi Y, Banerjee M, Mazis C, et al. Therapeutic Monoclonal Antibodies Targeting Immune Checkpoints for the Treatment of Solid Tumors. Antibodies (Basel) (2019) 8:51. doi: 10.3390/antib8040051

38. Pan C, Liu H, Robins E, Song W, Liu D, Li Z, et al. Next-generation immunooncology agents: current momentum shifts in cancer immunotherapy. $J$ Hematol Oncol (2020) 13:29. doi: 10.1186/s13045-020-00862-w

39. Nagasaki J, Togashi Y, Sugawara T, Itami M, Yamauchi N, Yuda J, et al. The critical role of CD4+ T cells in PD-1 blockade against MHC-II-expressing tumors such as classic Hodgkin lymphoma. Blood Adv (2020) 4:4069-82. doi: 10.1182/bloodadvances.2020002098

40. Li X, Wang R, Fan P, Yao X, Qin L, Peng Y, et al. A Comprehensive Analysis of Key Immune Checkpoint Receptors on Tumor-Infiltrating T Cells From Multiple Types of Cancer. Front Oncol (2019) 9:1066. doi: 10.3389/ fonc. 2019.01066

41. $\mathrm{He} \mathrm{X}, \mathrm{Xu} \mathrm{C}$. Immune checkpoint signaling and cancer immunotherapy. Cell Res (2020) 30:660-9. doi: 10.1038/s41422-020-0343-4

42. Marin-Acevedo JA, Soyano AE, Dholaria B, Knutson KL, Lou Y. Cancer immunotherapy beyond immune checkpoint inhibitors. J Hematol Oncol (2018) 11:8. doi: 10.1186/s13045-017-0552-6

43. Li X, Shao C, Shi Y, Han W. Lessons learned from the blockade of immune checkpoints in cancer immunotherapy. J Hematol Oncol (2018) 11:31. doi: 10.1186/s13045-018-0578-4

44. Tundo GR, Sbardella D, Lacal PM, Graziani G, Marini S. On the Horizon: Targeting Next-Generation Immune Checkpoints for Cancer Treatment. Chemotherapy (2019) 64:62-80. doi: 10.1159/000500902

45. Kudo M. Scientific Rationale for Combination Immunotherapy of Hepatocellular Carcinoma with Anti-PD-1/PD-L1 and Anti-CTLA-4 Antibodies. Liver Cancer (2019) 8:413-26. doi: 10.1159/000503254

46. Bose CK. Immune Checkpoint Blockers and Ovarian Cancer. Indian J Med Paediatr Oncol (2017) 38:182-9. doi: 10.4103/ijmpo.ijmpo_73_16
47. Cruz CR, Bollard CM. Adoptive Immunotherapy For Leukemia With Ex vivo Expanded T Cells. Curr Drug Targets (2017) 18:271-80. doi: 10.2174/ 1389450117666160209143529

48. Fan J, Shang D, Han B, Song J, Chen H, Yang JM. Adoptive Cell Transfer: Is it a Promising Immunotherapy for Colorectal Cancer? Theranostics (2018) 8:5784-800. doi: 10.7150/thno.29035

49. Sharpe M, Mount N. Genetically modified T cells in cancer therapy: opportunities and challenges. Dis Model Mech (2015) 8:337-50. doi: 10.1242/dmm.018036

50. Esmaeilzadeh A, Tahmasebi S, Athari SS. Chimeric antigen receptor -T cell therapy: Applications and challenges in treatment of allergy and asthma. BioMed Pharmacother (2020) 123:109685. doi: 10.1016/j.biopha.2019.109685

51. Wang Z, Wu Z, Liu Y, Han W. New development in CAR-T cell therapy. J Hematol Oncol (2017) 10:53. doi: 10.1186/s13045-017-0423-1

52. Fukuhara H, Ino Y, Todo T. Oncolytic virus therapy: A new era of cancer treatment at dawn. Cancer Sci (2016) 107:1373-9. doi: 10.1111/cas.13027

53. Cao GD, He XB, Sun Q, Chen S, Wan K, Xu X, et al. The Oncolytic Virus in Cancer Diagnosis and Treatment. Front Oncol (2020) 10:1786. doi: 10.3389/ fonc. 2020.01786

54. Pol J, Kroemer G, Galluzzi L. First oncolytic virus approved for melanoma immunotherapy. Oncoimmunology (2016) 5:e1115641. doi: 10.1080/ 2162402X.2015.1115641

55. Song M, Chen X, Wang L, Zhang Y. Future of anti-PD-1/PD-L1 applications: Combinations with other therapeutic regimens. Chin J Cancer Res (2018) 30:157-72. doi: 10.21147/j.issn.1000-9604.2018.02.01

56. Robert C. A decade of immune-checkpoint inhibitors in cancer therapy. Nat Commun (2020) 11:3801. doi: 10.1038/s41467-020-17670-y

57. Kalos M, June $\mathrm{CH}$. Adoptive $\mathrm{T}$ cell transfer for cancer immunotherapy in the era of synthetic biology. Immunity (2013) 39:49-60. doi: 10.1016/j.immuni.2013.07.002

58. Di S, Li Z. Treatment of solid tumors with chimeric antigen receptorengineered T cells: current status and future prospects. Sci China Life Sci (2016) 59:360-9. doi: 10.1007/s11427-016-5025-6

59. Thakar MS, Kearl TJ, Malarkannan S. Controlling Cytokine Release Syndrome to Harness the Full Potential of CAR-Based Cellular Therapy. Front Oncol (2019) 9:1529. doi: 10.3389/fonc.2019.01529

60. Groisberg R, Subbiah V. Immunotherapy and next-generation sequencing guided therapy for precision oncology: What have we learnt and what does the future hold? Expert Rev Precis Med Drug Dev (2018) 3:205-13. doi: 10.1080/ 23808993.2018.1480898

61. Nakagawa H, Fujita M. Whole genome sequencing analysis for cancer genomics and precision medicine. Cancer Sci (2018) 109:513-22. doi: $10.1111 /$ cas.13505

62. Peng M, Mo Y, Wang Y, Wu P, Zhang Y, Xiong F, et al. Neoantigen vaccine: an emerging tumor immunotherapy. Mol Cancer (2019) 18:128. doi: 10.1186/ s12943-019-1055-6

63. Tran E, Robbins PF, Rosenberg SA. 'Final common pathway' of human cancer immunotherapy: targeting random somatic mutations. Nat Immunol (2017) 18:255-62. doi: 10.1038/ni.3682

64. Yarchoan M, Johnson BA,3, Lutz ER, Laheru DA, Jaffee EM. Targeting neoantigens to augment antitumour immunity. Nat Rev Cancer (2017) 17:209-22. doi: 10.1038/nrc.2016.154

65. Wagner S, Mullins CS, Linnebacher M. Colorectal cancer vaccines: Tumorassociated antigens vs neoantigens. World J Gastroenterol (2018) 24:5418-32. doi: 10.3748 /wjg.v24.i48.5418

66. Sahin U, Tureci O. Personalized vaccines for cancer immunotherapy. Science (2018) 359:1355-60. doi: 10.1126/science.aar7112

67. Schumacher TN, Schreiber RD. Neoantigens in cancer immunotherapy. Science (2015) 348:69-74. doi: 10.1126/science.aaa4971

68. Zhao W, Wu J, Chen S, Zhou Z. Shared neoantigens: ideal targets for off-theshelf cancer immunotherapy. Pharmacogenomics (2020) 21:637-45 doi: $10.2217 /$ pgs-2019-0184

69. Klebanoff CA, Wolchok JD. Shared cancer neoantigens: Making private matters public. J Exp Med (2018) 215:5-7. doi: 10.1084/jem.20172188

70. Tureci O, Lower M, Schrors B, Lang M, Tadmor A, Sahin U. Challenges towards the realization of individualized cancer vaccines. Nat BioMed Eng (2018) 2:566-9. doi: 10.1038/s41551-018-0266-2

71. Fennemann FL, de Vries IJM, Figdor CG, Verdoes M. Attacking Tumors From All Sides: Personalized Multiplex Vaccines to Tackle Intratumor Heterogeneity. Front Immunol (2019) 10:824. doi: 10.3389/fimmu.2019.00824 
72. Whiteside TL, Demaria S, Rodriguez-Ruiz ME, Zarour HM, Melero I. Emerging Opportunities and Challenges in Cancer Immunotherapy. Clin Cancer Res (2016) 22:1845-55. doi: 10.1158/1078-0432.CCR-16-0049

73. Blass E, Ott PA. Advances in the development of personalized neoantigenbased therapeutic cancer vaccines. Nat Rev Clin Oncol (2021) 18:215-29. doi: 10.1038/s41571-020-00460-2

74. Richters MM, Xia H, Campbell KM, Gillanders WE, Griffith OL, Griffith M. Best practices for bioinformatic characterization of neoantigens for clinical utility. Genome Med (2019) 11:56. doi: 10.1186/s13073-019-0666-2

75. Roudko V, Greenbaum B, Bhardwaj N. Computational Prediction and Validation of Tumor-Associated Neoantigens. Front Immunol (2020) 11:27. doi: 10.3389/fimmu.2020.00027

76. Kosaloglu-Yalcin Z, Lanka M, Frentzen A, Logandha Ramamoorthy Premlal A, Sidney J, Vaughan K, et al. Predicting T cell recognition of MHC class I restricted neoepitopes. Oncoimmunology (2018) 7:e1492508. doi: 10.1080/ 2162402X.2018.1492508

77. Basith S, Manavalan B, Hwan Shin T, Lee G. Machine intelligence in peptide therapeutics: A next-generation tool for rapid disease screening. Med Res Rev (2020) 40:1276-314. doi: 10.1002/med.21658

78. Martins J, Magalhaes C, Rocha M, Osorio NS. Machine Learning-Enhanced T Cell Neoepitope Discovery for Immunotherapy Design. Cancer Inform (2019) 18:1176935119852081. doi: 10.1177/1176935119852081

79. Zhou C, Zhu C, Liu Q. Toward in silico Identification of Tumor Neoantigens in Immunotherapy. Trends Mol Med (2019) 25:980-92. doi: 10.1016/ j.molmed.2019.08.001

80. Kim S, Kim HS, Kim E, Lee MG, Shin EC, Paik S, et al. Neopepsee: accurate genome-level prediction of neoantigens by harnessing sequence and amino acid immunogenicity information. Ann Oncol (2018) 29:1030-6. doi: 10.1093/ annonc/mdy022

81. Wells DK, van Buuren MM, Dang KK, Hubbard-Lucey VM, Sheehan KCF, Campbell KM, et al. Key Parameters of Tumor Epitope Immunogenicity Revealed Through a Consortium Approach Improve Neoantigen Prediction. Cell (2020) 183:818-834 e813. doi: 10.1016/j.cell.2020.09.015

82. Kvistborg P, Clynes R, Song W, Yuan J. Immune monitoring technology primer: whole exome sequencing for neoantigen discovery and precision oncology. J ImmunoTher Cancer (2016) 4:22. doi: 10.1186/s40425-016-0126-0

83. Chen F, Zou Z, Du J, Su S, Shao J, Meng F, et al. Neoantigen identification strategies enable personalized immunotherapy in refractory solid tumors. J Clin Invest (2019) 129:2056-70. doi: 10.1172/JCI99538
84. Lopes A, Vandermeulen G, Preat V. Cancer DNA vaccines: current preclinical and clinical developments and future perspectives. J Exp Clin Cancer Res (2019) 38:146. doi: 10.1186/s13046-019-1154-7

85. Tran E, Turcotte S, Gros A, Robbins PF, Lu YC, Dudley ME, et al. Cancer immunotherapy based on mutation-specific CD4+ T cells in a patient with epithelial cancer. Science (2014) 344:641-5. doi: 10.1126/science.1251102

86. Tran E, Ahmadzadeh M, Lu YC, Gros A, Turcotte S, Robbins PF, et al. Immunogenicity of somatic mutations in human gastrointestinal cancers. Science (2015) 350:1387-90. doi: 10.1126/science.aad1253

87. Sahin U, Derhovanessian E, Miller M, Kloke BP, Simon P, Lower M, et al. Personalized RNA mutanome vaccines mobilize poly-specific therapeutic immunity against cancer. Nature (2017) 547:222-6. doi: 10.1038/nature23003

88. Ott PA, Hu Z, Keskin DB, Shukla SA, Sun J, Bozym DJ, et al. An immunogenic personal neoantigen vaccine for patients with melanoma. Nature (2017) 547:217-21. doi: 10.1038/nature22991

89. Parul A, Tulin D. 34th Annual Meeting \& Pre-Conference Programs of the Society for Immunotherapy of Cancer (SITC 2019): part 1 : National Harbor, MD, USA. 6-10 November 2019. J Immunother Cancer (2019) 7:282. doi: 10.1186/s40425-019-0763-1

90. Ott PA, Hu-Lieskovan S, Chmielowski B, Govindan R, Naing A, Bhardwaj N, et al. A Phase Ib Trial of Personalized Neoantigen Therapy Plus Anti-PD-1 in Patients with Advanced Melanoma, Non-small Cell Lung Cancer, or Bladder Cancer. Cell (2020) 183:347-362 e324. doi: 10.1016/j.cell.2020.08.053

91. Douglass J, Hsiue EH, Mog BJ, Hwang MS, DiNapoli SR, Pearlman AH, et al. Bispecific antibodies targeting mutant RAS neoantigens. Sci Immunol (2021) 6:eabd5515. doi: 10.1126/sciimmunol.abd5515

92. Hsiue EH, Wright KM, Douglass J, Hwang MS, Mog BJ, Pearlman AH, et al. Targeting a neoantigen derived from a common TP53 mutation. Science (2021) 371:eabc8697. doi: 10.1126/science.abc8697

Conflict of Interest: The authors declare that the research was conducted in the absence of any commercial or financial relationships that could be construed as a potential conflict of interest.

Copyright (C) 2021 Zhang, Lu, Qin, Gao, Tao, Su and Zhong. This is an open-access article distributed under the terms of the Creative Commons Attribution License (CC BY). The use, distribution or reproduction in other forums is permitted, provided the original author(s) and the copyright owner(s) are credited and that the original publication in this journal is cited, in accordance with accepted academic practice. No use, distribution or reproduction is permitted which does not comply with these terms. 